\section{International Scientific Journal Theoretical \& Applied Science}
p-ISSN: $2308-4944$ (print)
e-ISSN: 2409-0085 (online)
Year: 2016
Issue: 3
Volume: 35
Published: $30.03 .2016 \quad$ http://T-Science.org

SECTION 9. Chemistry and chemical technology.
Afsun Razzaq Sujayev

$\mathrm{PhD}$ in Chemistry, Senior Scientific Researcher Laboratory of Theoretical Bases of Synthesis and Action

Mechanism of Additives, Institute of Chemistry of Additives, Azerbaijan National Academy of Sciences, Azerbaijan s.afsun@mail.ru

Emin Nazir Garibov

$\mathrm{PhD}$ in Chemistry, Senior Scientific Researcher Laboratory of Theoretical Bases of Synthesis and Action Mechanism of Additives, Institute of Chemistry of Additives, Azerbaijan National Academy of Sciences, Azerbaijan e.garibov@yahoo.com

Vagif Majid Farzaliyev Doctor in Chemistry, Leading Researcher Laboratory of Theoretical Bases of Synthesis and Action Mechanism of Additives, Institute of Chemistry of Additives, Azerbaijan National Academy of Sciences, Azerbaijan aki05@mail.ru

\title{
SYNTHESIS AND TRANSFORMATIONS OF SOME TETRAHYDROPYRIMIDINE-5-CARBOXYLATES
}

Abstract: 2-(methacryloyloxy) ethyl 6-methyl-2-oxo-4-phenyl-1,2,3,4-tetrahydropyrimidine-5-carboxylate, which are cyclic ureas derivatives were synthesized from urea, 2-(methacryloyloxy) ethyl acetoacetate substituted and benzaldehyde.

Key words: Benzaldehyde; single-crystal X-ray study; urea; 2-(methacryloyloxy) ethyl acetoacetate.

Language: English

Citation: Sujayev AR, Garibov EN, Farzaliyev VM (2016) SYNTHESIS AND TRANSFORMATIONS OF SOME TETRAHYDROPYRIMIDINE-5-CARBOXYLATES. ISJ Theoretical \& Applied Science, 03 (35): 101106.

Soi: $\underline{\text { http://s-o-i.org/1.1/TAS-03-35-17 Doi: crossef http://dx.doi.org/10.15863/TAS.2016.03.35.17 }}$

\section{INTRODUCTION}

The pyrimidinethiones display many pharmacological properties, as part of our interest in this kind of materials, we report here the synthesis and crystal structure determination of the title compound ${ }^{[1]}$. Our synthesis is based in the Bidjinelli reaction, which consists on a three-component condensation of an aldehyde, a methylene active compound and an urea derivative in acidic media. This procedure is the most simple and useful for the preparation of 3,4-dihydropyrimidene-2 $(1 \mathrm{H})$ ones ${ }^{[2-4]}$.

New cyclic ureas were obtained by continuing researches in the field of the synthesis of various classes of organic nitrogen compounds and the study of their transformations. So that first time 2(methacryloyloxy)ethyl 6-methyl-2-oxo-4- phenyl-1,2,3,4-tetrahydropyrimidine-5carboxylate were obtained for the first time by us based on the trifluoroacetic acid catalist.

\section{EXPERIMENTAL}

Chemistry

Synthesis of 2-(methacryloyloxy) ethyl 6methyl-2-oxo-4-phenyl-1,2,3,4-

tetrahydropyrimidine-5-carboxylate (1)

Urea (1.20 gr, $0.02 \mathrm{~mol})$ is dissolved in 3:1 ratio of acetylacetone $(3 \mathrm{ml})$ and ethyl alcohol $\left(\begin{array}{ll}1 & \mathrm{ml}\end{array}\right)$ and 2-(methacryloyloxy)ethyl acetoacetate $(3.82 \mathrm{ml}, 0.02 \mathrm{~mol})$ is added on it drop by drop. After being dissolved in magnetic stirrer for 5 minutes benzaldehyde $(2.03 \mathrm{ml}$, $0.02 \mathrm{~mol}$ ) is added. After determining that the reaction has been fully completed, the solvent is evaporated. Processing of the reaction mixture was carried out by washing the reaction mixture 
with ice water, the precipitate was filtered, washed with $500 \mathrm{ml}$ of water, dried and recrystallized from ethanol $(75 \mathrm{ml})$. The yield is $2.4 \mathrm{~g}, \mathrm{mp} .211^{\circ} \mathrm{C}$.

Eluent-ethanol:hexane (5:2). ${ }^{1} \mathrm{H}$ NMR (300 MHz, DMSO-d6) 51.35 (s, 3H, $\mathrm{CH}_{3}$ ), 6.8-7.1 (m, H, Ar), 7.4 (m, H, Ar), 9.35 (s, $1 \mathrm{H}, \mathrm{NH}) .{ }^{13} \mathrm{C}$ NMR (75 MHz, DMSO-d6) 5 24, 29, 37, 51, 86, $117,122,129,132,141,151,205(\mathrm{C}=\mathrm{O})$.

Synthesis of allyl 1-(3-choloro-2hidroksipropyl)-4-metil-6-phenyl-2-thioxo1,2,3,6-tetrahydropyrimidine-5-carboxylate (2)

Allyl 6-methyl-4-phenyl-2-thioxo-1,2,3,4tetrahydropyrimidine-5-carboxylate $(0,908 \mathrm{gr}$, $0,0033 \mathrm{~mol}$ ) is dissolved in 2:1 ratio of acetylacetone $(10 \mathrm{ml})$ and ethyl alcohol $(5 \mathrm{ml})$ and epichlorohydrin $(0,26 \mathrm{ml}, 0,0033 \mathrm{~mol})$ is added on it drop by drop. After being dissolved in the stirrer for 25 minutes, 0.03 gr. aluminiumchloride catalyst is added on it and mixed by heating at $65-70^{\circ} \mathrm{C}$. The progress of the reaction is controlled by Sulifol UV 254 plate. After determining the full completion of reaction, solution is evaporated and is cleansed in ethyl alcohol solution. The white crystalline having the melting temperature of $168^{\circ} \mathrm{C}$ is obtained.

Eluent-ethanol:hexane (5:2). ${ }^{1} \mathrm{H}$ NMR (300 MHz, DMSO-d6) 2.30 (s, 3H, $\mathrm{CH}_{3}$ ), 7.06-7.25 $(\mathrm{m}, \mathrm{H}, \mathrm{Ar}), 4.81(\mathrm{H}, \mathrm{OH}), 9.84(\mathrm{~s}, 1 \mathrm{H}, \mathrm{NH})$, 3.40, $3.65\left(2 \mathrm{H}, \mathrm{CH}_{2}\right),{ }^{13} \mathrm{C}$ NMR $(75 \mathrm{MHz}$, DMSO-d6) 15.5, 49.5, 56,3, 67.8, 71.2, 74.5, $106,116.4,127.1,128.6,133.5,138,158.8$, 167.2, 178.4 .
Synthesis of ethyl 1-(2-hidroksibutyl)-4methyl-6-phenyl-2-thioxo-1,2,3,6tetrahydropyrimidine-5-carboxylate (3)

Ethyl 6-methyl-2-thioxo-4-(p-tolyl)-1,2,3,4tetrahydropyrimidine-5-carboxylate $(1.52 \mathrm{gr}$, $0.02 \mathrm{~mol})$ is dissolved in 2:1 ratio of acetylacetone $(12 \mathrm{ml})$ and ethyl alcohol $(5 \mathrm{ml})$ and 1,2-epoxobutane $(2.03 \mathrm{ml}, 0.02 \mathrm{~mol})$ is added on it drop by drop. After being dissolved in the stirrer for 30 minutes, 0.02 gr. aluminiumchloride catalyst is added on it and mixed by heating at $60-65^{\circ} \mathrm{C}$. The progress of the reaction is controlled by Sulifol UV 254 plate. After determining the full completion of reaction, solution is evaporated and is cleansed in ethyl alcohol solution. The white crystalline having the melting temperature of $192^{\circ} \mathrm{C}$ is obtained.

Eluent-ethanol:hexane (5:2). ${ }^{1} \mathrm{H}$ NMR (300 MHz, DMSO-d6) 0.96 (s, 3H, $\mathrm{CH}_{3}$ ), 4.59-7.25 $(\mathrm{m}, 6 \mathrm{H}, \mathrm{Ar}), 4.81(\mathrm{H}, \mathrm{OH}), 3.45(1 \mathrm{H}, \mathrm{CH}), 9.84$ (s, $1 \mathrm{H}, \mathrm{NH}), 3.40,1.48\left(2 \mathrm{H}, \mathrm{CH}_{2}\right) \cdot{ }^{13} \mathrm{C}$ NMR $(75$ MHz, DMSO-d6) 14.2, 28.6, 58.9, 61.7, 71.2, $71.6,104.2,127.1,127.9,128.6,129.9,138$, 160.3, 167.2, 178 .

\section{RESULTS AND DISCUSSION}

The synthesis of the new compounds is reported in Schemes 1, 2, 3. The reaction of substituted benzaldehyde with methylene active compounds such as 2-(methacryloyloxy)ethyl acetoacetate and and urea in the presence of trifluoroacetic acid led to the desired cyclic urea.<smiles>C=C(C)C(=O)OCCOC(=O)CC(C)=O</smiles>

Scheme 1

At the next stage we have provided the transformation of obtained compounds. So, by the reaction epichlorohydrin had synthesised allyl 1-(3-choloro-2-hidroksipropyl)-4-metil-6phenyl-2-thioxo-1,2,3,6-tetrahydropyrimidine5-carboxylate. 


\begin{tabular}{l|lrl|l|ll} 
& ISRA (India) & $=\mathbf{1 . 3 4 4}$ & SIS (USA) & $=\mathbf{0 . 9 1 2}$ & ICV (Poland) & $=\mathbf{6 . 6 3 0}$ \\
Impact Factor: & ISI (Dubai, UAE) $=\mathbf{0 . 8 2 9}$ & PUHU (Russia) $=\mathbf{0 . 1 7 9}$ & PIF (India) & $=\mathbf{1 . 9 4 0}$ \\
& GIF (Australia) & $\mathbf{0 . 5 6 4}$ & ESJI (KZ) & $=\mathbf{1 . 0 4 2}$ & IBI (India) & $=\mathbf{4 . 2 6 0}$
\end{tabular}<smiles>C=CCOC(=O)C1=C(C)NC(=S)NC1c1ccccc1</smiles>

Scheme 2

At the same time by the reaction 1,2epoxobutane synthesised ethyl 1-(2-<smiles>CCOC(=O)C1=C(C)NC(=S)NC1c1ccc(C)cc1</smiles><smiles>CCC1CO1</smiles><smiles>C1CCC1</smiles>

hidroksibutyl)-4-methyl-6-phenyl-2-thioxo1,2,3,6-tetrahydropyrimidine-5-carboxylate.

Scheme 3

The three-component condensation reactions come to an end within 2,5-3 hours at $60-75^{\circ} \mathrm{C}$. The synthesized compounds were crystalline and their structure was confirmed by spectral and physico-chemical methods, among which IQ, ${ }^{1} \mathrm{H}, \quad{ }^{13} \mathrm{C} \quad \mathrm{NMR}$ and $\mathrm{X}$-ray spectroscopy:

The crystal structure of synthesized 2(methacryloyloxy)ethyl 6-methyl-2-oxo-4- phenyl-1,2,3,4-tetrahydropyrimidine-5-

carboxylate has been determined with X-ray structure analysis method. The structure and crystalline form of new cyclical compound have been shown in the fig.1 and fig.2. Crystalline compound holds two crystallographical independent molecules.
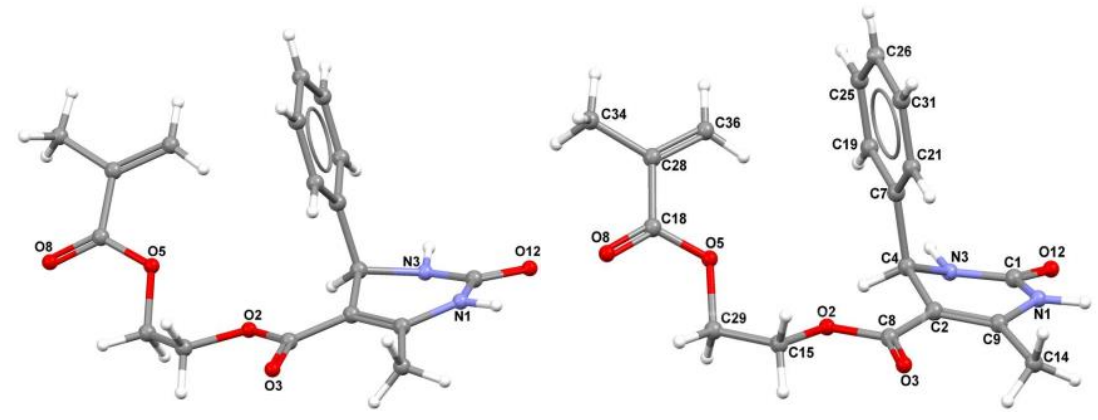

Figure 1 - The molecular structure of the new compound. 


\begin{tabular}{l|lrl|l|ll} 
& ISRA (India) & $=\mathbf{1 . 3 4 4}$ & SIS (USA) & $=\mathbf{0 . 9 1 2}$ & ICV (Poland) & $=\mathbf{6 . 6 3 0}$ \\
Impact Factor: & ISI (Dubai, UAE) $=\mathbf{0 . 8 2 9}$ & PUHU (Russia) $=\mathbf{0 . 1 7 9}$ & PIF (India) & $=\mathbf{1 . 9 4 0}$ \\
& GIF (Australia) & $\mathbf{0 . 5 6 4}$ & ESJI (KZ) & $=\mathbf{1 . 0 4 2}$ & IBI (India) & $\mathbf{4 . 2 6 0}$
\end{tabular}

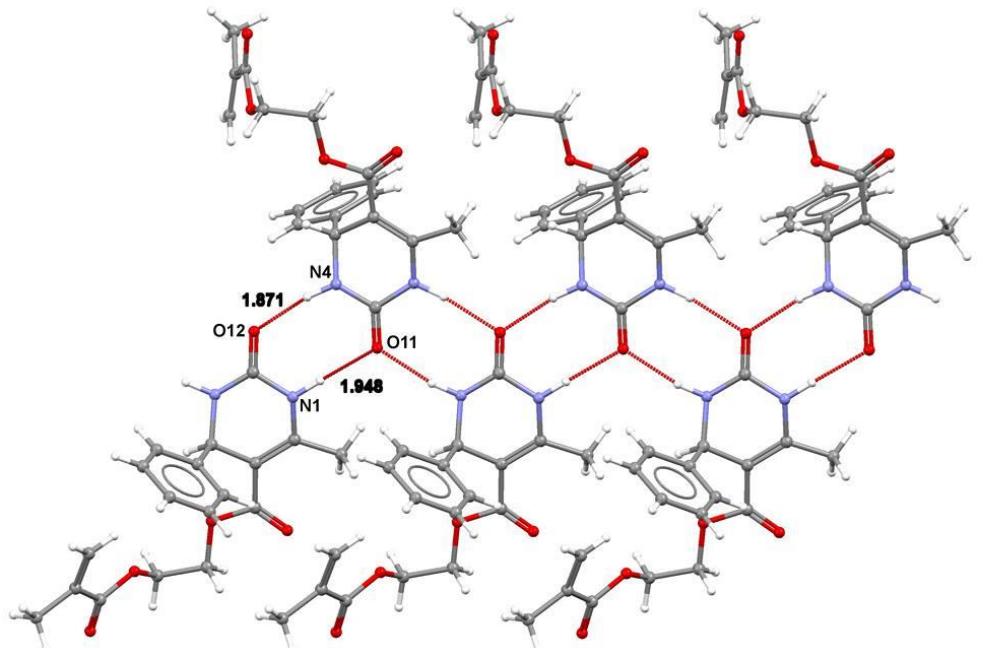

Figure 2 - Packing diagram of the title compound. N-H-O hydrogen bonds are shown as dashed lines. For clarity only one of the disordered components of the phenyl ring is shown.

In the compound, the $\mathrm{C} 8, \mathrm{C} 9, \mathrm{C} 11$ and $\mathrm{C} 12$ atoms of the phenyl ring are disordered over two sets of sites in a 0.60 (3):0.40 (3) ratio. The heterocycle ring is essentially planar and form a dihedral angle of with the phenyl ring. The crystal packing is stabilized by intermolecular N3-H3N $\cdots \mathrm{O} 1$ hydrogen bonds (Table 1-3), which link the molecules into chains running parallel to the $\mathrm{b}$ axis (Fig.2), with graph-set notation $\mathrm{C}(6)^{[5]}$.

Data collection: APEX2 ${ }^{[6]}$; cell refinement: SAINT-Plus [7]; data reduction: SAINT-Plus; program(s) used to solve structure: SHELXTL ${ }^{\text {[8- }}$ 9]; $\operatorname{program}(\mathrm{s})$ used to refine structure: SHELXTL; molecular graphics: SHELXTL; software used to preparematerial for publication: SHELXTL.

Table 1

\section{Crystal data and structure refinement for a_a.}

\begin{tabular}{llll}
\hline Identification code & $\mathrm{a} \_\mathrm{a}$ \\
Empirical formula & $\mathrm{C}_{18} \mathrm{H}_{20} \mathrm{~N}_{2} \mathrm{O}_{5}$ & \\
Formula weight & 344.36 & \\
Temperature & $373(2) \mathrm{K}$ & \\
Wavelength & $0.71073 \AA$ & $\mathrm{a}=90^{\circ}$. \\
Crystal system & Monoclinic & $\mathrm{b}=91.822(5)^{\circ}$. \\
Space group & $\mathrm{P} 21 / \mathrm{c}$ & $\mathrm{g}=90^{\circ}$. \\
Unit cell dimensions & $\mathrm{a}=16.441(4) \AA$ & \\
& $\mathrm{b}=27.869(6) \AA$ & \\
& $\mathrm{c}=7.1837(15) \AA$ & \\
Volume & $3289.8(12) \AA 3$ & \\
$Z$ & 8 & \\
Density (calculated) & $1.391 \mathrm{Mg} / \mathrm{m}^{3}$ & \\
Absorption coefficient & $0.102 \mathrm{~mm} \mathrm{~m}^{-1}$ & \\
F(000) & 1456 & \\
Crystal size & $0.12 \times 0.14 \mathrm{x} 0.18 \mathrm{~mm}^{3}$ & \\
Theta range for data collection & 1.916 to $26.998^{\circ}$. & \\
Index ranges & $-20<=\mathrm{h}<=20,-33<=\mathrm{k}<=35,-9<=1<=9$ \\
Reflections collected & 21576 & \\
Independent reflections & $6215[\mathrm{R}(\mathrm{int})=0.0544]$ & \\
Completeness to theta $=25.242^{\circ}$ & $92.7 \%$ & \\
Refinement method & Full-matrix least-squares on $\mathrm{F}^{2}$ \\
Data / restraints / parameters & $6215 / 0 / 455$ & \\
\hline
\end{tabular}




\begin{tabular}{l|lr|ll|ll} 
& ISRA (India) & $=\mathbf{1 . 3 4 4}$ & SIS (USA) & $=\mathbf{0 . 9 1 2}$ & ICV (Poland) & $=\mathbf{6 . 6 3 0}$ \\
Impact Factor: & ISI (Dubai, UAE) $=\mathbf{0 . 8 2 9}$ & PUHI (Russia) & $=\mathbf{0 . 1 7 9}$ & PIF (India) & $=\mathbf{1 . 9 4 0}$ \\
& GIF (Australia) & $=\mathbf{0 . 5 6 4}$ & ESJI (KZ) & $=\mathbf{1 . 0 4 2}$ & IBI (India) & $\mathbf{4 . 2 6 0}$ \\
& JIF & $=\mathbf{1 . 5 0 0}$ & SJIF (Morocco) & $\mathbf{2 . 0 3 1}$ & & \\
\hline
\end{tabular}

Goodness-of-fit on $\mathrm{F}^{2}$

Final $\mathrm{R}$ indices [I $>2 \operatorname{sigma}(\mathrm{I})]$

1.059

$\mathrm{R}$ indices (all data)

Extinction coefficient

$\mathrm{R}^{1}=0.0862, \mathrm{wR}^{2}=0.1890$

$\mathrm{R}^{1}=0.1206, \mathrm{wR}^{2}=0.2065$

Largest diff. peak and hole

$\mathrm{n} / \mathrm{a}$

0.491 and -0.311 e. $\AA^{-3}$

\section{Special details}

Geometry. All e.s.d.'s (except the e.s.d. in the dihedral angle between two 1.s. planes) are estimated using the full covariance matrix. The cell e.s.d.'s are taken into account individually in the estimation of e.s.d.'s in distances, angles and torsion angles; correlations between e.s.d.'s in cell parameters are only used when they are defined by crystal symmetry. An approximate (isotropic) treatment of cell e.s.d.'s is used for estimating e.s.d.'s involving l.s. planes.
Refinement. Refinement of $\mathrm{F}^{2}$ against ALL reflections. The weighted R-factor $w R$ and goodness of fit $S$ are based on $\mathrm{F}^{2}$, conventional R-factors $R$ are based on $\mathrm{F}$, with $F$ set to zero for negative $\mathrm{F}^{2}$. The threshold expression of $\mathrm{F}^{2}>$ $\sigma\left(\mathrm{F}^{2}\right)$ is used only for calculating R-factors (gt) etc. and is not relevant to the choice of reflections for refinement. R-factors based on $\mathrm{F}^{2}$ are statistically about twice as large as those based on $F$, and $R$ - factors based on ALL data will be even larger.

Table 2

Atomic coordinates $\left(x \mathbf{1 0}^{4}\right)$ and equivalent isotropic displacement parameters $\left(\AA^{2} \times 10^{3}\right)$ for a_a. $\mathrm{U}(\mathrm{eq})$ is defined as one third of the trace of the orthogonalized $\mathrm{U}^{\mathrm{ij}}$ tensor.

\begin{tabular}{|lrrrr|}
\hline & $\mathrm{x}$ & $\mathrm{y}$ & $\mathrm{z}$ & $\mathrm{U}(\mathrm{eq})$ \\
\hline $\mathrm{O}(1)$ & $4377(2)$ & $5866(1)$ & $2262(3)$ & $30(1)$ \\
$\mathrm{N}(1)$ & $8372(2)$ & $4725(1)$ & $11017(4)$ & $23(1)$ \\
$\mathrm{C}(1)$ & $7970(2)$ & $4850(1)$ & $9393(5)$ & $22(1)$ \\
$\mathrm{O}(2)$ & $10562(2)$ & $4190(1)$ & $7716(3)$ & $28(1)$ \\
$\mathrm{N}(2)$ & $6542(2)$ & $5330(1)$ & $5902(4)$ & $26(1)$ \\
$\mathrm{C}(2)$ & $9498(2)$ & $4417(1)$ & $9509(5)$ & $21(1)$ \\
$\mathrm{O}(3)$ & $10796(2)$ & $4197(1)$ & $10777(4)$ & $33(1)$ \\
$\mathrm{N}(3)$ & $8334(2)$ & $4734(1)$ & $7851(4)$ & $23(1)$ \\
$\mathrm{C}(3)$ & $6949(2)$ & $5210(1)$ & $4338(5)$ & $24(1)$ \\
$\mathrm{O}(4)$ & $4063(2)$ & $6419(1)$ & $-835(4)$ & $35(1)$ \\
$\mathrm{N}(4)$ & $6582(2)$ & $5331(1)$ & $2741(4)$ & $25(1)$ \\
$\mathrm{C}(4)$ & $8985(2)$ & $4381(1)$ & $7761(5)$ & $23(1)$ \\
$\mathrm{O}(5)$ & $10892(2)$ & $3631(1)$ & $4709(4)$ & $35(1)$ \\
$\mathrm{C}(5)$ & $5744(2)$ & $5472(1)$ & $5859(5)$ & $24(1)$ \\
$\mathrm{O}(6)$ & $4073(2)$ & $5793(1)$ & $5246(4)$ & $38(1)$ \\
$\mathrm{C}(6)$ & $4563(2)$ & $5770(1)$ & $4061(5)$ & $27(1)$ \\
$\mathrm{O}(7)$ & $3104(2)$ & $6969(1)$ & $-1087(4)$ & $46(1)$ \\
$\mathrm{C}(7)$ & $8654(2)$ & $3884(1)$ & $7352(5)$ & $26(1)$ \\
$\mathrm{O}(8)$ & $11800(2)$ & $3283(1)$ & $2941(4)$ & $50(1)$ \\
$\mathrm{C}(8)$ & $10340(2)$ & $4261(1)$ & $9474(5)$ & $24(1)$ \\
$\mathrm{C}(9)$ & $9167(2)$ & $4569(1)$ & $11078(5)$ & $22(1)$ \\
$\mathrm{C}(10)$ & $6314(2)$ & $6189(1)$ & $2500(5)$ & $30(1)$ \\
$\mathrm{O}(11)$ & $7599(2)$ & $4997(1)$ & $4428(3)$ & $25(1)$ \\
$\mathrm{C}(11)$ & $5949(2)$ & $5696(1)$ & $2635(5)$ & $25(1)$ \\
$\mathrm{O}(12)$ & $7324(2)$ & $5070(1)$ & $9420(3)$ & $27(1)$ \\
$\mathrm{C}(12)$ & $3796(3)$ & $6859(1)$ & $-1188(5)$ & $32(1)$ \\
$\mathrm{C}(13)$ & $5338(2)$ & $5431(1)$ & $7662(5)$ & $28(1)$ \\
$\mathrm{C}(14)$ & $9574(2)$ & $4601(1)$ & $12942(5)$ & $28(1)$ \\
$\mathrm{C}(15)$ & $535(2)$ & $3984(2)$ & $7530(6)$ & $33(1)$ \\
$\mathrm{C}(16)$ & $5415(2)$ & $5634(1)$ & $4262(5)$ & $26(1)$ \\
\hline
\end{tabular}




\begin{tabular}{|c|c|c|c|c|c|c|}
\hline Impact Factor: & $\begin{array}{l}\text { ISRA (India) } \\
\text { ISI (Dubai, UAE } \\
\text { GIF (Australia) } \\
\text { JIF }\end{array}$ & $\begin{array}{l}=1.344 \\
=0.829 \\
=0.564 \\
=1.500\end{array}$ & $\begin{array}{l}\text { SIS (USA) } \\
\text { PИНЦ (Russia) } \\
\text { ESJI (KZ) } \\
\text { SJIF (Morocco) }\end{array}$ & $\begin{array}{l}=0.912 \\
=0.179 \\
=1.042 \\
=2.031\end{array}$ & $\begin{array}{l}\text { ICV (Poland) } \\
\text { PIF (India) } \\
\text { IBI (India) }\end{array}$ & $\begin{array}{l}=6.630 \\
=1.940 \\
=4.260\end{array}$ \\
\hline
\end{tabular}

\begin{tabular}{|lrrrr|}
\hline $\mathrm{C}(17)$ & $3566(2)$ & $6026(1)$ & $1842(5)$ & $29(1)$ \\
$\mathrm{C}(18)$ & $11114(3)$ & $3339(2)$ & $3364(6)$ & $36(1)$ \\
$\mathrm{C}(19)$ & $8189(2)$ & $3812(2)$ & $5739(6)$ & $34(1)$ \\
$\mathrm{C}(20)$ & $6721(2)$ & $6317(2)$ & $912(6)$ & $34(1)$ \\
$\mathrm{C}(21)$ & $8791(3)$ & $3504(2)$ & $8498(6)$ & $37(1)$ \\
$\mathrm{C}(22)$ & $6300(3)$ & $6520(1)$ & $3921(6)$ & $37(1)$ \\
$\mathrm{C}(23)$ & $3490(2)$ & $6073(2)$ & $-210(6)$ & $33(1)$ \\
$\mathrm{C}(24)$ & $7103(2)$ & $6755(2)$ & $797(7)$ & $40(1)$ \\
$\mathrm{C}(25)$ & $7872(2)$ & $3372(2)$ & $5320(6)$ & $41(1)$ \\
$\mathrm{C}(26)$ & $8008(3)$ & $2994(2)$ & $6490(7)$ & $43(1)$ \\
$\mathrm{C}(27)$ & $4449(3)$ & $7190(2)$ & $-1704(6)$ & $38(1)$ \\
$\mathrm{C}(28)$ & $10421(3)$ & $3088(2)$ & $2481(6)$ & $41(1)$ \\
$\mathrm{C}(29)$ & $11501(2)$ & $3939(2)$ & $5515(6)$ & $36(1)$ \\
$\mathrm{C}(30)$ & $6686(3)$ & $6950(2)$ & $3801(7)$ & $47(1)$ \\
$\mathrm{C}(31)$ & $8474(3)$ & $3062(2)$ & $8070(7)$ & $47(1)$ \\
$\mathrm{C}(32)$ & $4224(3)$ & $7688(2)$ & $-1877(6)$ & $47(1)$ \\
$\mathrm{C}(33)$ & $7095(3)$ & $7069(2)$ & $2246(7)$ & $47(1)$ \\
$\mathrm{C}(34)$ & $10619(4)$ & $2765(2)$ & $962(8)$ & $73(2)$ \\
$\mathrm{C}(35)$ & $5203(3)$ & $7024(2)$ & $-1952(7)$ & $57(1)$ \\
$\mathrm{C}(36)$ & $9671(3)$ & $3154(2)$ & $3056(6)$ & $43(1)$ \\
\hline
\end{tabular}

Hydrogen bonds for a_a $\left[\AA ̊\right.$ and $\left.{ }^{\circ}\right]$.

Table 3

\begin{tabular}{|c|c|c|c|c|}
\hline D-H...A & $\mathrm{d}(\mathrm{D}-\mathrm{H})$ & $\mathrm{d}(\mathrm{H} . . . \mathrm{A})$ & $\mathrm{d}(\mathrm{D} \ldots \mathrm{A})$ & $<(\mathrm{DHA})$ \\
\hline $\mathrm{N}(1)-\mathrm{H}(1) \ldots \mathrm{O}(11) \# 1$ & 0.95 & 1.95 & $2.897(4)$ & 176.4 \\
\hline $\mathrm{N}(2)-\mathrm{H}(2) \ldots \mathrm{O}(12)$ & 0.95 & 1.95 & $2.890(4)$ & 171.8 \\
\hline $\mathrm{N}(3)-\mathrm{H}(3) \ldots \mathrm{O}(11)$ & 0.95 & 1.90 & $2.802(4)$ & 157.8 \\
\hline $\mathrm{N}(4)-\mathrm{H}(4) \ldots \mathrm{O}(12) \# 2$ & 0.95 & 1.87 & $2.809(4)$ & 169.1 \\
\hline $\mathrm{C}(14)-\mathrm{H}(14 \mathrm{~A}) \ldots \mathrm{O}(3)$ & 0.96 & 2.32 & $2.814(5)$ & 111.0 \\
\hline $\mathrm{N}(1)-\mathrm{H}(1) \ldots \mathrm{O}(11) \# 1$ & 0.95 & 1.95 & $2.897(4)$ & 176.4 \\
\hline $\mathrm{N}(2)-\mathrm{H}(2) \ldots \mathrm{O}(12)$ & 0.95 & 1.95 & $2.890(4)$ & 171.8 \\
\hline $\mathrm{N}(3)-\mathrm{H}(3) \ldots \mathrm{O}(11)$ & 0.95 & 1.90 & $2.802(4)$ & 157.8 \\
\hline $\mathrm{N}(4)-\mathrm{H}(4) \ldots \mathrm{O}(12) \# 2$ & 0.95 & 1.87 & $2.809(4)$ & 169.1 \\
\hline $\mathrm{C}(14)-\mathrm{H}(14 \mathrm{~A}) \ldots \mathrm{O}(3)$ & 0.96 & 2.32 & $2.814(5)$ & 111.0 \\
\hline
\end{tabular}

\section{References:}

1. Dalinger D, Stadler A, Kappe CO (2004) Pure Appl. Chem. 76, 10171024.

2. Kotharkar SA, Nagawade RR, Shinde DB (2006) UkrainicaBioorg. Acta, 2, pp.17-21.

3. Lu J, Bai Y, Wang Z, Yang B, Ma H (2000) Tetrahedron Lett. 41, 90759078.

4. Salehi P, Davizi M, Zolfigol AM, Fard MA (2003) Tetrahedron Lett.44, pp.2889-289.

5. Bernstein J, Davis RE, Shimoni L, Chang NL (1995) Hydrogen-bond pattern functionality and graph sets, (1995). Angew. Chem. Int. Ed. Engl. 34, pp.1555-1573.

6. Bruker (2001) SAINT-Plus. Bruker AXS, Madison, Wisconsin, USA.

7. Bruker (2005) APEX2. Bruker AXS, Madison, Wisconsin, USA.

8. Sheldrick GM (2003) SADABS. Bruker AXS, Madison, Wisconsin, USA. Sheldrick, G. M.(2008). Acta Cryst. A64, pp.112-122.

9. Srinivas KV, Das B (2004) Synthesis, 13, pp.2091-2093. 